\title{
Gravitational Theory without the Cosmological
}

\section{Constant Problem, Symmetries of Space-Filling}

\author{
Branes and Higher Dimensions
}

\author{
E.I.Guendelman*and A.B.Kaganovich ${ }^{\dagger}$ \\ Physics Department, Ben Gurion University of the Negev, Beer Sheva \\ 84105, Israel
}

\begin{abstract}
We showed that the principle of nongravitating vacuum energy, when formulated in the first order formalism, solves the cosmological constant problem. The most appealing formulation of the theory displays a local symmetry associated with the arbitrariness of the measure of integration. This can be motivated by thinking of this theory as a direct coupling of physical degrees of freedom with a "space - filling brane" and in this case such local symmetry is related to space-filling brane gauge invariance. The model is formulated in the first

*GUENDEL@BGUmail.BGU.AC.IL

†ALEXK@BGUmail.BGU.AC.IL
\end{abstract}


order formalism using the metric $G_{A B}$ and the connection $\Gamma_{B C}^{A}$ as independent dynamical variables. An additional symmetry (Einstein - Kaufman symmetry) allows to eliminate the torsion which appears due to the introduction of the new measure of integration. The most successful model that implements these ideas is realized in a six or higher dimensional space-time. The compactification of extra dimensions into a sphere gives the possibility of generating scalar masses and potentials, gauge fields and fermionic masses. It turns out that remaining four dimensional space-time must have effective zero cosmological constant. 


\section{Introduction}

We have developed a theory [1], [2] where the measure of integration in the action principle is not necessarily $\sqrt{-G}\left(G=\operatorname{Det}\left(G_{A B}\right)\right)$ but it is determined dynamically through additional degrees of freedom. This theory is based on the demand that such measure respect the principle of non gravitating vacuum energy (NGVE principle) which states that the Lagrangian density $L$ can be changed to $L+$ constant without affecting the dynamics. This requirement is imposed in order to offer a new approach for the solution of the cosmological constant problem [3].

Clearly the invariance $L \longrightarrow L+$ constant for the action is achieved if the measure of integration in the action is a total derivative, so that to an infinitesimal hypercube in D-dimensional space-time $x_{0}^{A} \leq x^{A} \leq x_{0}^{A}+d x^{A}, A=0,1, \ldots, D-1$ we associate a volume element $d V$ which is: (i) a total derivative, (ii) it is proportional to $d^{D} x$ and (iii) $d V$ is a general coordinate invariant. The usual choice, $\sqrt{-G} d^{D} x$ does not satisfy condition (i).

All of the conditions (i)-(iii) are satisfied [1], [2] if the measure appropriate to the integration in the space of $D$ scalar fields $\varphi_{a},(a=1,2, \ldots D)$, that is

$$
d V=d \varphi_{1} \wedge d \varphi_{2} \wedge \ldots \wedge d \varphi_{D} \equiv \frac{\Phi}{D !} d^{D} x
$$

where

$$
\Phi \equiv \varepsilon_{a_{1} a_{2} \ldots a_{D}} \varepsilon^{A_{1} A_{2} \ldots A_{D}}\left(\partial_{A_{1}} \varphi_{a_{1}}\right)\left(\partial_{A_{2}} \varphi_{a_{2}}\right) \ldots\left(\partial_{A_{D}} \varphi_{a_{D}}\right)
$$

Notice that this is a particular realization of the coupling of $p$-brane (with $p+1=$ 
$D)$ with the $(p+1)$-form potential

$$
A_{A_{1} A_{2} \ldots A_{D}}=\partial_{\left[A_{1}\right.} A_{\left.A_{2} \ldots A_{D}\right]}
$$

and a further coupling with the Lagrangian density (usually not considered). In fact, if $A_{A_{2} \ldots A_{D}}$ equals to $A_{A_{2} \ldots A_{D}}^{(\varphi)} \equiv \frac{1}{D !} \varphi_{a_{1}}\left(\partial_{A_{2}} \varphi_{a_{2}}\right) \ldots\left(\partial_{A_{D}} \varphi_{a_{D}}\right) \varepsilon_{a_{1} a_{2} \ldots a_{D}}$, then $\partial_{\left[A_{1}\right.} A_{\left.A_{2} \ldots A_{D}\right]} d x^{A_{1}} \wedge$ $d x^{A_{2}} \wedge \ldots \wedge d x^{A_{D}}$ coincides with (1), (2).

Following the Ref. [4] we will call the brane a "space-filling brane" if the rank of the form $A_{A_{1} A_{2} \ldots A_{D}}$ that couples to the p-brane equals to the space-time dimensionality (that is $p+1=D$ ). In the normal formulation of $p$-branes one requires invariance under gauge transformations of the form

$$
A_{A_{1} A_{2} \ldots A_{D}} \longrightarrow A_{A_{1} A_{2} \ldots A_{D}}+\partial_{\left[A_{1}\right.} \Lambda_{\left.A_{2} \ldots A_{D}\right]}
$$

and simply write the coupling $g \int A_{A_{1} A_{2} \ldots A_{D}} d x^{A_{1}} \wedge d x^{A_{2}} \wedge \ldots \wedge d x^{A_{D}}$ which is invariant under (四) provided $\Lambda_{\left[A_{2} \ldots A_{D}\right]} \rightarrow 0$ as $x^{A} \rightarrow \infty$ and one doesn't allow coupling to a third entity (like the Lagrangian density $L$ ).

The problem is [4] that in the case of a "space-filling brane" the equation of motion obtained from varying $A_{A_{1} A_{2} \ldots A_{D}}$ is simply $g=0$, that is there is no action principle to talk about.

In the alternative we propose, we don't have to necessarily insist on the particular realization (11),(2), although it has the most attractive geometrical interpretation [2].

We will choose to write $A_{A_{1} A_{2} \ldots A_{D}}$ as a total derivative

$$
A_{A_{1} A_{2} \ldots A_{D}}=\partial_{\left[A_{1}\right.} A_{\left.A_{2} \ldots A_{D}\right]}
$$


and then we use $A_{A_{2} \ldots A_{D}}$ as the independent dynamical variables in our action principle. Furthermore we can implement the NGVE-principle if we write the following action

$$
S=\int L \partial_{\left[A_{1}\right.} A_{\left.A_{2} \ldots A_{D}\right]} d x^{A_{1}} \wedge d x^{A_{2}} \wedge \ldots \wedge d x^{A_{D}}
$$

which describes the coupling of the brane to gravity and matter which appear through the Lagrangian density $L=L_{g}+L_{m}$. The Lagrangian structure has to be defined by the demand that the action (6) be invariant under the following gauge transformation

$$
A_{A_{2} \ldots A_{D}} \longrightarrow A_{A_{2} \ldots A_{D}}+\Lambda_{A_{2} \ldots A_{D}}
$$

for any $\Lambda_{A_{2} \ldots A_{D}}$ (without a condition for $\Lambda_{A_{2} \ldots A_{D}}$ as $x^{A} \rightarrow \infty$ ) which will be refered afterwards as a "space-filling brane gauge transformation". In this case $L$ has to transform correspondingly in order to compensate the transformation of the measure. How this is realized, will be explained after we understand the basic structure of the theory in the first order formalism (see also Ref. [2]). In the case that we use the representation (1),(2), an arbitrary change of the measure corresponds to an arbitrary diffeomorphism in the internal space of the scalar fields $\varphi_{a}$.

There are two well known variational principles: the first and the second order formalisms, which are equivalent in the case of the general theory of relativity. However, as we will see, they are inequivalent in our case. In the first order formalism, in the action $G_{A B}$ and $\Gamma_{B C}^{A}$ appear, while no explicit derivatives of $G_{A B}$ are introduced in the Lagrangian density. The action principle allows then to solve $\Gamma_{B C}^{A}$ as a function of $G_{A B}$ and its first derivatives. The resulting equations are the usual Einstein equations which are also obtained from the second order formalism which does not 
involve $\Gamma_{B C}^{A}$ as a dynamical variable but rather involves only $G_{A B}$ and their first and second derivatives.

In the case at hand, that is in the context of the NGVE theories, the first and second order formalisms are not equivalent. The model that results from studying the theory in the second order formalism [1 gives rise to empty space solutions with arbitrary constant curvature. In this case the cosmological constant problem is not solved (although arguments based on maximal symmetry can be made in favor of the zero curvature choice for vacuum). In contrast, the first order formalism leads to the solution of the cosmological constant problem in a straightforward way.

In the first order formalism, the theory has been studied [2] using the vielbein $e_{i}^{A}$ and the spin-connection $\omega_{i k}^{A}(i, k$ denote Lorentz indexes in $\mathrm{D}$ dimensions), instead of utilizing $\Gamma_{B C}^{A}$ which will be the case here. Furthermore, the use of $\Gamma_{B C}^{A}$ as dynamical variables instead of $\omega_{i k}^{A}$ makes manifest a new symmetry of the theory, which was discovered as a symmetry of the curvature tensor in the affine connection space by Einstein and Kaufman long time ago [5] and given by them the name of " $\lambda$-transformation". Although the $\lambda$-symmetry was discussed in Ref.[5] in the context of a very specific unified model, it turns out that the range of applicability of this symmetry is much wider. This question will be discussed in Sec.2.

The importance of Einstein-Kaufman $\lambda$-symmetry in our model is that it allows for the elimination of the torsion in the absence of fermions, as opposed with the first order formalism employing $\omega_{i k}^{A}$ where it is hard to avoid explicitly the appearence of the torsion even in the absence of fermions [2]. 
In our previous paper [2] it was shown that in the first order formalism, the theory based on the NGVE-principle possesses an additional local symmetry for the vacuum and for some special models. When realizing the NGVE-principle with the measure of the form of eqs.(11),(2), we have seen[2] that this local symmetry incorporates the group of diffeomorphism transformations of the internal space of scalar fields $\varphi_{a}$. Here we will see that this local symmetry can be formulated in a way where it incorporates space-filling brane gauge invariance (7)(see sections 3-5 of this paper). The importance of this symmetry, apart from its obvious geometrical meaning, consists of the fact that for models where it holds it is possible to choose the gauge where the mea-

sure $\Phi$ coincides with the measure of general relativity $\sqrt{-G}$. This is why we call this symmetry "local Einstein symmetry". In Sec.6 we construct realistic models (without loosing the solution of the cosmological constant problem in four dimensions) where the local Einstein symmetry holds as an exact symmetry.

\section{Action and Einstein-Kaufman $\lambda$-symmetry}

According to the NGVE-principle, the total action in the D-dimensional space-time should be written in the form

$$
S=\int \Phi L d^{D} x
$$

where $\Phi d^{D} x$ may be given either by

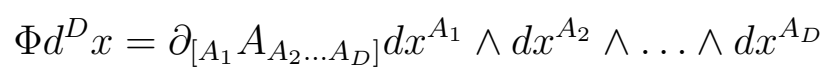

(as in eq.(6)), or by using $\Phi$ as in eqs.(11),(2). 
We assume that $L$ does not contain the measure fields, that is the fields by means of which $\Phi$ is defined. If this condition is satisfied then the theory has an additional symmetry. In fact, for example for the case of the action with $\Phi$ given by eq.(2), the action (8) is invariant under the infinitesimal shift of the fields $\varphi_{a}$ by an arbitrary infinitesimal function of the total Lagrangian density $L$, that is [1], [2]

$$
\varphi_{a}^{\prime}=\varphi_{a}+\epsilon g_{a}(L), \epsilon \ll 1
$$

Our choice for the total Lagrangian density is

$$
L=-\frac{1}{\kappa} R(\Gamma, G)+L_{m}
$$

where $L_{m}$ is the matter Lagrangian density and $R(\Gamma, G)$ is the scalar curvature

$$
\begin{gathered}
R(\Gamma, G)=G^{A B} R_{A B}(\Gamma) \\
R_{A B}(\Gamma)=R_{A B C}^{C}(\Gamma) \\
R_{B C D}^{A}(\Gamma) \equiv \Gamma_{B C, D}^{A}-\Gamma_{B D, C}^{A}+\Gamma_{E D}^{A} \Gamma_{B C}^{E}-\Gamma_{E C}^{A} \Gamma_{B D}^{E}
\end{gathered}
$$

The curvature tensor is invariant under the $\lambda$ - transformation

$$
\Gamma_{B C}^{\prime A}=\Gamma_{B C}^{A}+\delta_{B}^{A} \lambda, C
$$

which was discovered by Einstein and Kaufman[5]. Although this symmetry was discussed in Ref. [5] in the very specific unified theory, it turns out that $\lambda$-symmetry has a wider range of validity and in particular it is useful in our case. 
In fact, for a wide class of matter models, the matter Lagrangian density $L_{m}$ is invariant under the $\lambda$ transformation too. This is obvious if $L_{m}$ does not include the connection $\Gamma_{B C}^{A}$ at all (like, for example, for scalar fields, for a point particle and other cases that we will discuss in this paper). As an example of particular importance we consider here the case of Dirac fermions in 4-dimensional space-time with the hermitian Lagrangian density

$$
L_{f}=-\frac{i}{2}\left[\left(\nabla_{\mu} \bar{\psi}\right) \gamma^{\mu} \psi-\bar{\psi} \gamma^{\mu} \nabla_{\mu} \psi+2 i V(\bar{\psi} \psi)\right]
$$

which is also invariant under $\lambda$-transformation. Here matrices $\gamma^{\mu}(\mu=0,1,2,3)$ are defined according to $\gamma^{\mu}=e_{n}^{\mu} \gamma^{n}$, where $\gamma^{n}$ are the Dirac matrices and $e_{n}^{\mu}$ are vielbeins: $G^{\mu \nu}=e_{n}^{\mu} e^{n \nu}$. The covariant derivatives in (16) are given by $\nabla_{\mu} \psi=\partial_{\mu} \psi-\frac{1}{4} \Gamma_{\mu \nu \lambda} \gamma^{\nu} \gamma^{\lambda} \psi$, $\nabla_{\mu} \bar{\psi}=\partial_{\mu} \bar{\psi}-\frac{1}{4} \Gamma_{\mu \nu \lambda} \bar{\psi} \gamma^{\nu} \gamma^{\lambda}$ and $\Gamma_{\mu \nu \lambda}=G_{\lambda \sigma} \Gamma_{\mu \nu}^{\sigma}$.

What concerns with vector bosons, we note that the demand of gauge invariance leads to a generally coordinate invariant gauge boson Lagrangian which does not include the connection [7].

\section{Connection and local symmetries}

First consider here the case where $L_{m}$ does not depend on $\Gamma_{B C}^{A}$, that is fermions and curvature are not present in $L_{m}$. Varying the action (8), (11) with respect to $\Gamma_{B C}^{A}$, we get

$$
-\Gamma_{B C}^{A}-\Gamma_{E B}^{D} G^{E A} G_{D C}+\delta_{C}^{A} \Gamma_{B D}^{D}+\delta_{B}^{A} G^{D E} \Gamma_{D E}^{F} G_{F C}-
$$




$$
G_{D C} \partial_{B} G^{D A}+\delta_{B}^{A} G_{D C} \partial_{E} G^{D E}-\delta_{C}^{A} \frac{\Phi, B}{\Phi}+\delta_{B}^{A} \frac{\Phi, C}{\Phi}=0
$$

We will look for the solution (up to a $\lambda$-symmetry transformation) of the form

$$
\Gamma_{B C}^{A}=\left\{\begin{array}{l}
A \\
B C
\end{array}\right\}+\Sigma_{B C}^{A}
$$

where $\left\{\begin{array}{l}A \\ B C\end{array}\right\}$ are the Christoffel's connection coefficients. Then $\Sigma_{B C}^{A}$ satisfies equation

$$
-\sigma,_{C} G_{A B}+\sigma,{ }_{A} G_{B C}-G_{B D} \Sigma_{C A}^{D}-G_{A D} \Sigma_{B C}^{D}+G_{A B} \Sigma_{C D}^{D}+G_{B C} G_{D A} G^{E F} \Sigma_{E F}^{D}=0
$$

where

$$
\sigma \equiv \ln \chi, \quad \chi \equiv \frac{\Phi}{\sqrt{-g}}
$$

The general solution of eq. (19) is

$$
\Sigma_{B C}^{A}=\delta_{B}^{A} \lambda,,_{C}+\frac{1}{D-2}\left(\sigma,{ }_{B} \delta_{C}^{A}-\sigma,_{D} G_{B C} G^{A D}\right)
$$

where $\lambda$ is an arbitrary function, which appears due to the existence of the EinsteinKaufman $\lambda$-symmetry. If we choose the gauge $\lambda=\sigma /(D-2)$, then the antisymmetric part of $\Sigma_{B C}^{A}$ disappears and we get finally

$$
\Sigma_{B C}^{A}(\sigma)=\frac{1}{D-2}\left(\delta_{B}^{A} \sigma,,_{C}+\delta_{C}^{A} \sigma,_{B}-\sigma_{, D} G_{B C} G^{A D}\right)
$$

In the presence of fermions, for the case $D=4$, in addition to the $\sigma$-dependent contribution to the connection(18), there is the usual fermionic contribution $\Sigma_{B C}^{(f) A}$ which does not depend on $\sigma$ (see for example Ref.[6]). However, even in the presence of fermions we can use the $\lambda$-transformation since $L_{f}$ (see eq.(16)) is invariant under the $\lambda$-transformation. Due to this, the $\sigma$-dependent contribution to the antisymmetric part of $\Sigma_{B C}^{A}$ can be set to zero also here. Therefore we can write

$$
\Sigma_{B C}^{A}=\Sigma_{B C}^{A}(\sigma)+\Sigma_{B C}^{(f) A}
$$


where $\Sigma_{B C}^{A}(\sigma)$ is again defined by eq.(22).

In the vacuum, the $\sigma$-contribution (22) to the connection can be eliminated by a conformal transformation of the metric [8] accompanied by a corresponding transformation of the fields defining the measure $\Phi$. Indeed, in the vacuum the action (8),(11) is invariant under local transformations

$$
\begin{gathered}
G_{A B}(x)=J^{-1} G_{A B}^{\prime}(x) \\
\Phi(x)=J^{-1}(x) \Phi^{\prime}(x)
\end{gathered}
$$

For $J=\chi^{2 /(D-2)}$ we get $\chi^{\prime} \equiv 1, \Sigma_{B C}^{A}(\sigma) \equiv 0$ and $\Gamma_{B C}^{A}=\left\{{ }_{B C}^{A}\right\}^{\prime}$, where $\left\{{ }_{B C}^{A}\right\}^{\prime}$ are the Christoffel's coefficients corresponding to the new metric $G_{A B}^{\prime}$. The appropriate generalization of the local symmetry $(24), 25)$ in the presence of fermions will be discussed in Sec. 5. The extension of applicability of this local symmetry for realistic matter models will be discussed in Sec.6.

For the case where the measure $\Phi$ is given by eq.(2), the transformation (25) can be the result of a diffeomorphism $\varphi_{a} \longrightarrow \varphi_{a}^{\prime}=\varphi_{a}^{\prime}\left(\varphi_{b}\right)$ in the space of the scalar fields $\varphi_{a}$ (see Ref. [2]). Then $J=\operatorname{Det}\left(\frac{\partial \varphi_{a}^{\prime}}{\partial \varphi_{b}}\right)$.

If we take the choice (9), then eq.(25) for a given $J$ may be interpreted as the result of the gauge transformation (77).

\section{Equations of motion}

First we study equations that originate from the variation with respect to the measure fields. If the measure is defined using the antisymmetric tensor field $A_{A_{2} \ldots A_{D}}$ as the 
dynamical variable, we obtain

$$
\epsilon^{A_{1} \ldots A_{D}} \partial_{A_{D}}\left[-\frac{1}{\kappa} R(\Gamma, G)+L_{m}\right]=0
$$

which means that

$$
-\frac{1}{\kappa} R(\Gamma, G)+L_{m}=M=\text { constant }
$$

If we consider the case where the measure is defined as in eq.(2), we obtain instead of (26), the equation

$$
A_{b}^{B} \partial_{B}\left[-\frac{1}{\kappa} R(\Gamma, G)+L_{m}\right]=0
$$

where $A_{b}^{B}=\varepsilon_{a_{1} \ldots a_{D-1} b} \varepsilon^{A_{1} \ldots A_{D-1} B}\left(\partial_{A_{1}} \varphi_{a_{1}}\right) \ldots\left(\partial_{A_{D-1}} \varphi_{a_{D-1}}\right)$. Since $A_{b}^{A} \partial_{A} \varphi_{b^{\prime}}=D^{-1} \delta_{b b^{\prime}} \Phi$ it follows that $\operatorname{Det}\left(A_{b}^{A}\right)=\frac{D^{-D}}{D !} \Phi^{D-1}$, so that if $\Phi \neq 0$, eq.(27) is again obtained.

Therefore the two approaches for defining the measure which implements the NGVE principle, give, under regular conditions, the same equation (that is eq.(27)). The case where the measure is defined as in eq.(2), provides with an extra possibility, which is that (27) may not be satisfied if $\Phi=0$. That is one can envision a scenario where the integration constant $M$ in eq.(27) could change while going through a singular surface with $\Phi=0$. This possibility and its cosmological consequences will be studied in a separate work.

Let us now study equations that originate from variation with respect to $G^{A B}$. For simplicity we present here the calculations for the case where there are no fermions. Performing the variation with respect to $G^{A B}$ we get

$$
-\frac{1}{\kappa} R_{A B}(\Gamma)+\frac{\partial L}{\partial G^{A B}}=0
$$


Contracting eq.(29) with $G^{A B}$ and making use eq.(27) we get the constraint

$$
G^{A B} \frac{\partial\left(L_{m}-M\right)}{\partial G^{A B}}-\left(L_{m}-M\right)=0
$$

This constraint has to be satisfied for all components (in the functional space) of the function $L_{m}$. In particular, for the constant part denoted $<L_{m}>$, which is relevant to a maximally symmetric vacuum state, we get

$$
<L_{m}>-M=0
$$

Inserting (31) in eq.(27) we see that in the maximally symmetric vacuum the scalar curvature $R(\Gamma)$ is equal to zero. As we have seen in the previous section, the $\sigma$-contribution to the connection can be eliminated in the vacuum by the transformations (24), 25) (notice that due to the NGVE-principle, the constant part of the matter Lagrangian density $<L_{m}>$ does not alter the result that the action (8) in the vacuum is invariant under the transformations (24),(25). This is because the measure $\Phi$ is a total derivative and therefore constant part of the Lagrangian density does not contribute into equations of motion). Then in terms of the new metric $G_{A B}^{\prime}$, the scalar curvature $R(\Gamma, G)$ becomes the usual scalar curvature $R\left(G_{A B}^{\prime}\right)$ of the Riemannian space-time with the metric $G_{A B}^{\prime}$. Therefore we conclude that the Riemannian scalar curvature vanishes in the maximally symmetric vacuum. In the presence of fermions the constraint (30) has to be generalized. For more details about fermionic models see the next section. 


\section{Some matter models which satisfy automatically the constraint (30) and local Einstein symmetry}

As we have seen, the consistency of the equations of motions demands the constraint

(30) to be satisfied. Here we are going to present theories where the constraint (30) is associated with the existence of a local symmetry, which we have already identified in the vacuum case, i.e. the symmetry $(24),(25)$, which is associated with space-filling brane gauge invariance or with diffeomorphism invariance of the internal space of the fields $\varphi_{a}$. The model in the absence of this symmetry can also make sense[2], but then the geometrical interpretation of the theory is lost (in this case the constraint can still hold, but then the symmetry degrees of freedom becomes physical). Therefore, in what follows we will discuss cases when the local symmetry $(24),(25)$ holds (possibly appropriately generalized) even when matter fields are introduced (we called this symmetry "local Einstein symmetry").

The following examples satisfy the local Einstein symmetry and constraint (30) (the cases of gauge fields, massive scalar fields and massive fermions will be discussed in Sec.6).

1.Scalar fields without potentials, including fields subjected to non linear constraints, like the $\sigma$ model[1], [2]. The general coordinate invariant action for these cases has the form $S_{m}=\int L_{m} \Phi d^{D} x$ where $L_{m}=\frac{1}{2} \sigma,_{A} \sigma,_{B} g^{A B}$.

2.Matter consisting of fundamental bosonic strings[1], [2]. The constraint (30) can be verified by representing the string action in the $D$-dimensional form where $G_{A B}$ 
plays the role of a background metric. For example, bosonic strings, according to our formulation, where the measure of integration in a $D$ dimensional space-time is chosen to be $\Phi d^{D} x$, will be governed by an action of the form:

$$
\begin{aligned}
S_{m} & =\int L_{\text {string }} \Phi d^{D} x, \\
L_{\text {string }} & =-T \int d \sigma d \tau \frac{\delta^{D}(x-X(\sigma, \tau))}{\sqrt{-G}} \sqrt{\operatorname{Det}\left(G_{A B} X_{, a}^{A} X_{, b}^{B}\right)}
\end{aligned}
$$

where $\int L_{\text {string }} \sqrt{-G} d^{D} x$ would be the action of a string embedded in a $D$-dimensional space-time in the standard theory; $a, b$ label coordinates in the string world sheet and $T$ is the string tension. Notice that under a transformation (24), $L_{\text {string }} \rightarrow$ $J^{(D-2) / 2} L_{\text {string }}$, therefore concluding that $L_{\text {string }}$ is a homogeneous function of $G^{A B}$ of degree one, that is constraint(30) is satisfied only if $D=4$.

3.It is possible [1], [2] to formulate the point particle model of matter in four dimensions $(D=4)$ in a way such that eq.(30) is satisfied. This is because for the free falling point particle a variety of actions are possible (and are equivalent in the context of general relativity). The usual actions in the 4-dimensional space-time with the metric $g_{\mu \nu}$ are taken to be $S=-m \int F(y) d s$, where $y=g_{\mu \nu} \frac{d X^{\mu}}{d s} \frac{d X^{\nu}}{d s}$ and $s$ is determined to be an affine parameter except if $F=\sqrt{y}$, which is the case of reparametrization invariance. In our model we must take $S_{m}=-m \int L_{\text {part }} \Phi d^{4} x$ with $L_{\text {part }}=-m \int d s \frac{\delta^{4}(x-X(s))}{\sqrt{-g}} F(y(X(s)))$ where $\int L_{p a r t} \sqrt{-g} d^{4} x$ would be the action of a point particle in 4 dimensions in the usual theory. For the choice $F=y$, constraint (30) is satisfied. Unlike the case of general relativity, different choices of $F$ lead to unequivalent theories.

4.In the presence of Dirac fermions with the Lagrangian density (16) (in four 
dimensions, $D=4$ ) the local Einstein symmetry (24),(25) is appropriately generalized to

$$
\begin{gathered}
e_{\mu}^{a}(x)=J^{-1 / 2}(x) e_{\mu}^{\prime a}(x) ; e_{a}^{\mu}(x)=J^{1 / 2}(x) e_{a}^{\prime \mu}(x) \\
\Phi(x)=J^{-1}(x) \Phi^{\prime}(x) \\
\psi(x)=J^{1 / 4}(x) \psi^{\prime}(x) ; \bar{\psi}(x)=J^{1 / 4}(x) \bar{\psi}^{\prime}(x)
\end{gathered}
$$

provided that $V(\bar{\psi} \psi) \propto(\bar{\psi} \psi)$ or $\left(\bar{\psi} \gamma_{i} \psi\right)\left(\bar{\psi} \gamma^{i} \psi\right)$, which describe a Nambu - JonaLasinio type interaction [9]. Notice that in this case the condition for the invariance of the action with the matter Lagrangian (16) under the transformations (34-(36) is not just the simple homogeneity of degree 1 in $g^{\mu \nu}$ or degree 2 in $e_{a}^{\mu}$, because of the presence of the fermion transformation (36). However, the invariance under (34)-(36) together with the fermionic equations of motion gives now the constraint in the form

$$
e^{a \mu} \frac{\partial L_{m}}{\partial e^{a \mu}}-2 L_{m}=0
$$

This constraint was discussed in Ref. [2] without reference to the generalized local Einstein symmetry (34)-(36). From the results of Sec.3 concerning the $\lambda$-symmetry of the fermionic term of the action (see eq.(23)) and making use the local Einstein symmetry (34)-(36) we can reduce the connection to the usual one in the presence of fermions [6]. 


\section{Gauge fields, scalar fields with nontrivial poten- tials and massive fermions from a six dimen- sional theory}

We have seen in the previous paper[2] that when trying to introduce gauge fields into the theory in a way which is consistent with the local Einstein symmetry(24),(25), this runs against the problem that the gauge field kinetic energy $G^{A B} G^{C D} F_{A C} F_{B D}$ has homogeneity of degree 2 in $G^{A B}$ instead of degree 1 which is needed in order to satisfy the constraint (30). We have shown also in Ref. [2] how this problem can be avoided in the framework of the Kaluza-Klein approach. However, the solution of this problem suggested in Ref.[2] seems to be not realistic enough.

We now will show how it is possible to construct more realistic models then those discussed before, by working in the context of a higher dimensional theory with two or more compactified dimensions with curvature. In this case we can introduce curvature dependence in prefactors of gauge field kinetic energy, scalar field potentials or fermionic mass such that the local Einstein symmetry be an exact symmetry.

In this case we consider an action of the form (the case of fermions will be considered at the end of this section)

$$
S=\int \Phi d^{6} x\left[-\frac{1}{\kappa} R(\Gamma, G)-\frac{\lambda}{R(\Gamma, G)} F_{A B} F^{A B}+\frac{1}{2} G^{A B} \partial_{A} \varphi \partial_{B} \varphi-R(\Gamma, G) V(\varphi)\right],
$$

where $F_{A B} \equiv \partial_{A} A_{B}-\partial_{B} A_{A}$. The prefactors $\lambda / R(\Gamma, G)$ in the gauge field kinetic energy and $R(\Gamma, G)$ in the scalar field potential $V(\varphi)$ are required so as to preserve 
the local Einstein symmetry (24), (25).

The simplest realization of this idea is achieved in a six dimensional model where two dimensions are compactified into a sphere. We will see that the models we discuss allow and seem to prefer this type of compactification. Furthermore, for solutions which are maximally symmetric in the remaining four dimensions, the noncompactified 4-dimensional space-time is only Minkowski space. This means that starting from a higher dimensional model we achieve a four dimensional solution of the cosmological constant problem.

The simplest model that respects the local Einstein symmetry and gives rise to $M^{4} \times S^{2}$ compactified solution, is a model where compactification is triggered by a non linear sigma model.

In this case

$$
S=\int \Phi d^{6} x\left[-\frac{1}{\kappa} R(\Gamma, G)+\frac{1}{2} G^{A B} \partial_{A} \vec{\phi} \cdot \partial_{B} \vec{\phi}\right]
$$

where the scalar field $\vec{\phi}$ is an isovector constrained to satisfy $\vec{\phi}^{2}=f^{2}=$ constant. This model is invariant under the local Einstein symmetry(24),(25).

For the hedgehog configuration

$$
\vec{\phi}=f(\cos \theta, \sin \theta \sin \varphi, \sin \theta \cos \varphi)
$$

the $M^{4} \times S^{2}$ metric

$$
d s^{2}=-d t^{2}+d \vec{x}^{2}+b^{2}\left(d \theta^{2}+\sin ^{2} \theta d \varphi^{2}\right)
$$

where $b$ is an arbitrary constant, is a solution in the gauge $\chi=1$ (that is in the gauge where the gravitational equations coincide with the 6-dimensional Einstein's 
equations) provided $f^{2}=2 / \kappa$ (see Ref.[10]). If one wants to avoid the fine tuning of this parameter of the Lagrangian one can use instead a no scale non linear sigma model where the size of the surface in isospin space is determined dynamically [11). In this case $b$ is not determined by the equations of motion. The $M^{4} \times S^{2}$ form of compactification can be seen quite directly from the form of the equations $R_{A B}=$ $\kappa \frac{\partial L}{\partial G^{A B}}$, since for the case (40), (41) we immediately obtain the condition $R_{\mu \nu}=0$ $(\mu, \nu=0,1,2,3)$ and $R_{\varphi}^{\varphi}+R_{\theta}^{\theta}=R=2 / b^{2} \neq 0$. Finally, we should point out that the possible Kaluza-Klein gauge fields acquire a big mass in this case[12], so they don't appear in the low energy physics.

It is interesting to see that it is possible to induce $M^{4} \times S^{2}$ compactification from a gauge-field monopole configuration in the extra dimensions $S^{2}$. Let us consider the first two terms of the action (38), i.e. the action describing gravity + gauge fields in a locally Einstein symmetric way. For the magnetic monopole $A_{A}=0$ if $A=0,1,2,3$, $A_{\theta}=0, A_{\varphi}=m(\cos \theta \mp 1)$ and if

$$
\begin{array}{r}
d s^{2}=g_{\mu \nu} d x^{\mu} d x^{\nu}+b^{2}(x) d \Omega^{2} \\
d \Omega^{2}=d \theta^{2}+\sin ^{2} \theta d \varphi^{2}, \\
\mu, \nu=0,1,2,3,
\end{array}
$$

we find the equations

$$
\begin{gathered}
\frac{1}{\kappa} R_{\mu \nu}(\Gamma)=\lambda \frac{R_{\mu \nu}(\Gamma)}{R^{2}(\Gamma, G)} F^{2} \\
\frac{1}{\kappa} R_{a b}(\Gamma)=\lambda \frac{R_{a b}(\Gamma)}{R^{2}(\Gamma, G)} F^{2}-\frac{2 \lambda}{R(\Gamma, G)} F_{a c} F_{b}^{c},(a, b=\theta, \varphi)
\end{gathered}
$$

where $F^{2}=F_{A B} F^{A B}$. 
If $R_{\mu \nu}(\Gamma) \neq 0$, then eqs.(43), (44) imply $F^{2}=0$, which is not consistent with the monopole ansatz. Using that

$$
R_{\mu \nu}(\Gamma)=0
$$

we see that $R(\Gamma, G)=R_{\theta}^{\theta}+R_{\varphi}^{\varphi}$ and from eq.(44) we get

$$
R^{2}(\Gamma, G)=-\kappa \lambda F^{2}
$$

Notice that the action (38) respects the $\lambda$-symmetry. Due to the local Einstein symmetry of the action, we can again fix the gauge where $\chi \equiv 1$ (that is $\Phi \equiv \sqrt{-g}$ ). Then the $\sigma$-contribution (22) to the connection is equal to zero.

When working with the action of Sec.3, we were able to find the connection $\Gamma_{B C}^{A}$ as a solution of eq.(17) without using the equations of motion which follow from the variation with respect to $G^{A B}$. Now however the scalar curvature enters in the equation obtained from the variation with respect to $\Gamma_{B C}^{A}$. Therefore we have to solve these equations together.

We are interested now in solutions which are maximally symmetric with respect to the remaining four dimensions. Therefore in (42) we choose $g_{\mu \nu}(x)$ as a metric of a maximally symmetric 4-dimensional space-time with 10 Killing vectors and $b(x)=$ constant. Then $F^{2}$ is a constant. In this case, the variation with respect to $\Gamma_{B C}^{A}$ leads again to an equation like (17) with a common factor $\left(-\frac{1}{\kappa}+\frac{\lambda}{R^{2}(\Gamma, G)} F^{2}\right)$. It follows from eq. (46), that this factor is not equal to zero. Therefore, in the case under consideration, the magnetic monopole configuration does not contribute to the connection and hence the solution of eq.(17) is now just the Christoffel's connection coefficients: $\Gamma_{B C}^{A}=\left\{\begin{array}{l}A \\ B C\end{array}\right\}$. It means that $R_{A B}(\Gamma)$ is just the usual Ricci tensor 
$R_{A B}\left(G_{C D}\right)$. Therefore eq.(15) takes the form $R_{\mu \nu}\left(g_{\lambda \sigma}\right)=0$ where $R_{\mu \nu}\left(g_{\lambda \sigma}\right)$ is the usual Ricci tensor of the four dimensional space-time with a maximally symmetric metric $g_{\lambda \sigma}$. We conclude that the very curved extra dimensions are necessarily accompanied only with the flat maximally symmetric four dimensional space-time, that is with Minkowski space.

Equation (46) with $R=R\left(G_{A B}\right)$ gives us the value of the strength of the magnetic

monopole: $m=\sqrt{2 / \kappa \lambda}$. Notice that the constant size $b$ of the extra dimensions is not determined. The existence of this flat direction is associated (from the 4-dimensional point of view) with a massless scalar field. Whether this is a phenomenological problem depends on the coupling and possible cosmological evolution of such scalar field. This will be studied in the future.

In this model there is no mass generation for the Kaluza-Klein gauge fields, which can therefore play a role in the low energy physics. It is also interesting to notice that what matter does in the extra dimensions produces directly curvature only in the extra dimensional space, at least in the ground state. That is, there is no mixing between Planck scale physics and low energy physics.

This can be compared with the well known Freund-Rubin compactification, for example when applied to 11-dimensional supergravity[13], where an expectation value of a four index field strength $F_{A B C D}$ in four dimensions is responsible for curving four dimensions into an anti de Sitter space and also for the compactification of seven dimensions into a sphere, i.e., a complete mix up of the physics of compactification and the physics that dominates the large scale structure of the observed four dimensions. 
With the addition of a potential of the form $R(\Gamma, G) V(\varphi)$ (consistent with the local Einstein symmetry) to eq.(38), the possibility of a scalar field with non trivial dynamics and in particular the possibility of mass for the scalar field and of spontaneous symmetry breaking appears in a straightforward fashion. Notice that phase transitions associated with a change of $\langle V(\phi)>$ correspond to a change in the effective Newton constant and not related to a change of vacuum energies, which cannot enter into the theory anyway.

Let us consider now the possibility of fermionic mass generation in 4-dimensional space-time in a way consistent with the local Einstein symmetry. If we look for a term which generates a fermionic mass term as a result of compactification in the form $f R^{n} \bar{\psi} \psi$ with dimensionless coupling constant $f$, then $n$ has to be equal to 1/2. In this case the only space-time dimension $D$ which allows the local Einstein symmetry (34)-(36) is $D=6$. Therefore for the fermionic mass generation in 4dimensional space-time (without introduction new dimensionful coupling constant) we have to start from the 6 -dimensional model with the action

$$
S_{f}=-\frac{i}{2} \int \Phi d^{6} x\left[\left(\nabla_{A} \bar{\psi}\right) \gamma^{A} \psi-\bar{\psi} \gamma^{A} \nabla_{A} \psi+2 i \sqrt{R(\Gamma, G)} \bar{\psi} \psi\right]
$$

For $\gamma$-matrices and other quantities in six dimensions see Ref. [14. After the compactification of two extra dimensions into a sphere, the curvature of the sphere induces a mass for fermions in 4-dimensional space-time. 


\section{Discussion}

We have shown that the NGVE-principle in the context of the first order formalism solves the cosmological constant problem. In this paper we have formulated several models (in the above framework) that respect the local Einstein symmetry which has nice geometrical interpretations. Furthermore, in models where the local Einstein symmetry is the exact symmetry, we always have both constraint(30) and possibility to obtain the measure $\sqrt{-G}$ by setting the gauge $\Phi=\sqrt{-G}$.

Using higher dimensional $(D \geq 6)$ models it is possible to maintain this local Einstein symmetry while constructing realistic models which allow for gauge fields, mass generation, spontaneous symmetry breaking, etc.. This is possible to realize in the presence of compactification of extra dimensions into a sphere and simultaneously achieving zero 4-dimensional cosmological constant. This result is related to the fact that in such a model the physics that is responsible for compactification does not affect the geometry of the large scale structure of the uncompactified four dimensional space-time.

Furthermore, in the case where we use the gauge model (38), compactification appears not only as a choice, since the alternative six-dimensional maximally symmetric vacuum with $R=0$ would be a sick vacuum. This is not only because $1 / R$ is undefined but also because the small perturbations bring us to the region where the gauge field kinetic term has wrong sign which is of course an unstable regime.

Finally, if it is the case that the local Einstein symmetry can be maintained even after quantum corrections are considered, we get an interesting constraint on the form 
of the possible quantum corrections which can be only terms homogeneous of degree 1 in $G^{A B}$ like for example $R_{A B} R^{A B} / R$.

\section{Acknowledgements}

We would like to thank N.Kaloper for interesting conversations. 


\section{References}

[1] E.I. Guendelman and A.B. Kaganovich, Phys. Rev. D53, 7020 (1996). See also E.I. Guendelman and A.B. Kaganovich, in:Proceedings of the third Alexander Friedmann International Seminar on Gravitation and Cosmology, edited by Yu.N.Gnedin, A.A. Grib, V.M. Mostepanenko (Friedman Laboratory Publishing, St Petersburg, 1995).

[2] E.I. Guendelman and A.B. Kaganovich, "Gravitational Theory without the Cosmological Constant Problem", Phys. Rev.D, 1997, to appear; gr-qc/9611046.

[3] S.Weinberg, Rev. Mod. Phys. 61, 1 (1989); Y.J. Ng, Int. J. Mod. Phys. D1, 145, (1992); Gravitation and Modern Cosmology, The Cosmological Constant Problem, edited by A.Zichichi, V. de Sabbata and N. Sanchez (Ettore Majorana International Science Series, Plenum Press, 1991). For the history of the problem see for example discussion in : I. Novikov, Evolution of the Universe, Cambridge University Press, 1983.

[4] Y.Polchinski, Phys.Rev.Lett. 75,4724 (1995).

[5] A.Einstein, The Meaning of Relativity, Fifth Edition, MJF Books, N.Y.1956; see Appendix II, which according to a note by Einstein on the fifth edition, reports on joint work with B.Kaufman.

[6] F.W.Hehl and B.K.Datta, Journ. of Math. Phys. 12, 1334 (1971).

[7] F.W.Hehl, P.von der Heyde and G.D.Kerlik, Rev. Mod. Phys. 48, 393 (1976). 
[8] N.D.Birrell and P.C.W.Davies, Quantum fields in curved space. Cambridge University Press, Cambridge, 1984.

[9] Y.Nambu and G.Jona-Lasinio, Phys. Rev. 122, 345 (1961).

[10] E.Cremmer and J.Sherk, Nucl. Phys.B108, 409 (1979); C.Omero and R.Percacci, Nucl. Phys. B165, 351 (1980); M.Gell-Mann and B.Zwiebach, Phys. Lett.147B, 111 (1984); E.Cremmer and J.Sherk, Nucl. Phys. B118, 61 (1977); G.Clemment, Class. Quantum Grav.5, 325 (1988); J.F.Luciani, Nucl. Phys. B135, 11 (1978); M.Gell-Mann and B.Zwiebach, Nucl. Phys. B260, 569 (1985).

[11] E.I.Guendelman, Phys. Rev. D50, 7538 (1994); E.I.Guendelman, Class. Quantum Grav. 12, 1893 (1995).

[12] M. Gell-Mann and B. Zwiebach, Phys. Lett. 141B, 333 (1984).

[13] P.G.O.Freund and M.A.Rubin, Phys.Lett. 97B, 233 (1980).

[14] S.Randjbar-Daemi, Abdus Salam, J.Strathdee, Nucl.Phys. 214, 491 (1983). Reprinted in: Modern Kaluza-Klein Theories, Ed. by T.Appelquist, A.Chodos and P.G.O.Freund, Addison-Wesley Publishing Company, Inc., 1987. 